\title{
Health Policy and Practice
}

Galied S. R. Muradin, MD

M. G. Myriam Hunink, MD, $\mathrm{PhD}$

Index terms:

Arteries, extremities, 92.721

Arteries, grafts and prostheses, 92.1268

Arteriosclerosis, 92.721

Cost-effectiveness

Interventional procedures,

comparative studies, 92.126,

92.128

Technology assessment

Radiology 2001; 218:464-469

Abbreviations:

$\mathrm{NHB}=$ net health benefit

PTA = percutaneous transluminal angioplasty

QALY = quality-adjusted life-year

${ }^{1}$ From the Program for the Assessment of Radiological Technology (ART Program), Departments of Radiology (G.S.R.M., M.G.M.H.) and Epidemiology and Biostatistics (M.G.M.H.), Erasmus University Medical Center Rotterdam, Rm EE21-40a, Dr. Molewaterplein 50, 3015 GE Rotterdam, the Netherlands; and the Department of Health Policy and Management, Harvard School of Public Health, Boston, Mass (M.G.M.H.). Received January 10, 2000; revision requested March 4; revision received April 25; accepted June 1. Address correspondence to M.G.M.H. (e-mail: hunink@epib.fgg.eur $. n I)$.

○ RSNA, 2001

\section{Author contributions:}

Guarantor of integrity of entire study, M.G.M.H.; study concepts, study design, and definition of intellectual content, G.S.R.M., M.G.M.H.; literature research, G.S.R.M.; data acquisition, G.S.R.M., M.G.M.H.; data analysis, G.S.R.M.; statistical analysis, G.S.R.M., M.G.M.H.; manuscript preparation, G.S.R.M.; manuscript editing, review, and final version approval, G.S.R.M., M.G.M.H.

\section{Cost and Patency Rate Targets for the Development of Endovascular Devices to Treat Femoropopliteal Arterial Disease ${ }^{1}$}

PURPOSE: To determine the criteria that would make use of an endovascular device cost-effective compared with bypass surgery and percutaneous transluminal angioplasty in the treatment of femoropopliteal arterial disease.

MATERIALS AND METHODS: A decision model was developed to compare treatment with the use of a hypothetical endovascular device with established therapies. Cost-effectiveness from the perspective of the health care system was considered. Outcome measures were lifetime costs and quality-adjusted life-years. With the use of net health benefit calculations and threshold analysis, combinations of costs and patency rates were determined that would make the device cost-effective compared with established therapies. In subgroup and sensitivity analyses, the effect on decision-making of sex, age, indication, lesion type, procedural risk, and society's willingness to pay for incremental gain in health were explored.

RESULTS: Use of a device that costs $\$ 3,000$ would be cost-effective compared with bypass surgery for critical ischemia if the 5 -year patency rate is $29 \%-46 \%$. Use of the same device would be cost-effective compared with angioplasty for disabling claudication and stenosis if the 5 -year patency rate is $69 \%-86 \%$.

CONCLUSION: The target combinations of costs and patency rates found in this study are probably attainable, and further development of such endovascular devices seems warranted.

Although bypass surgery and percutaneous transluminal angioplasty (PTA) are commonly used revascularization procedures in the treatment of femoropopliteal arterial occlusive disease, both procedures have disadvantages (1). Percutaneous transluminal balloon angioplasty is a low-risk and low-cost procedure, but it is associated with a fairly high restenosis rate (2-5). Primary stent placement does not improve the patency rate of PTA performed for femoropopliteal arterial disease and is thus currently used only to salvage a failed balloon angioplasty procedure $(6,7)$. Bypass surgery, on the other hand, has higher long-term patency rates but is also associated with a higher procedural risk, higher cost, and longer convalescence period $(4,5)$. In general, PTA is performed as primary treatment of short focal lesions of the femoropopliteal artery, whereas bypass surgery is the primary treatment in diffuse disease.

Endovascular devices are currently being developed as alternative interventions to overcome the problems of established procedures. Important considerations in choosing the optimal treatment strategy are the effectiveness of the device, the risks of the procedures, and the costs. These parameters are generally unknown during the development of a new technology. In particular, the patency and cost estimates associated with endovascular devices are uncertain and may even change with time. It is difficult, if not impossible, to predict what the precise values for the parameters of a new technology will be. Given 


\begin{tabular}{|c|c|c|}
\hline Parameter & Bypass Surgery & Angioplasty \\
\hline \multicolumn{3}{|l|}{ Procedural mortality (\%) } \\
\hline Claudication & 0.8 & 0.2 \\
\hline Critical ischemia & 4.7 & 3.2 \\
\hline Procedural nonfatal systemic morbidity (\%) & 8.5 & 1.3 \\
\hline Time lost due to convalescence (d) & 7 & 2 \\
\hline \multicolumn{3}{|l|}{ Procedural cost ${ }^{*}$} \\
\hline Claudication & 20,531 & 10,168 \\
\hline Critical ischemia & 25,881 & 18,171 \\
\hline \multicolumn{3}{|l|}{ Primary patency, 1-y/5-y (\%) } \\
\hline Claudication and stenosis & $91 / 80$ & $79 / 68$ \\
\hline Claudication and occlusion & $91 / 80$ & $53 / 35$ \\
\hline Critical ischemia and stenosis & $84 / 66$ & $62 / 47$ \\
\hline Critical ischemia and occlusion & $84 / 66$ & $28 / 12$ \\
\hline
\end{tabular}

the outcome and cost of established procedures, however, we can calculate under what conditions a new technology can become cost-effective compared with the established procedures, thereby setting standards for the new device. The merit of such an approach is that it can potentially focus the development of new technology $(8,9)$. This applies not only to femoropopliteal interventions but also to many other procedures such as the use of an abdominal aortic endoprosthesis and placement of a carotid stent.

The purpose of this study was to determine the criteria that would make the use of an endovascular device for the treatment of femoropopliteal arterial disease cost-effective compared with PTA and bypass surgery. A secondary objective was to illustrate how decision and cost-effectiveness analysis can be used to focus the development of new technologies.

\section{MATERIALS AND METHODS}

\section{Overview Decision Model}

Previously, a decision analytic model was developed to examine the choice between bypass surgery and PTA for femoropopliteal arterial occlusive disease (10). The model was used to consider different types of patients (age, sex, other risk factors), varying severity of disease (disabling claudication, rest pain, tissue loss), and different types of lesions (stenosis, occlusion) and to combine literature data on risks, benefits, and costs. The main outcome measures were quality-adjusted life expectancy and lifetime cost for each strategy, depending on patient characteristics, clinical indication, and lesion type.
In the current study, another treatment option was added to the model, namely, treatment with a hypothetical endovascular device. We compared three treatment strategies. Each strategy allowed at most two treatments. Initial revascularization was accomplished with balloon angioplasty, with autologous saphenous vein bypass surgery, or use of the hypothetical endovascular device. Secondary treatment for primary failure was undertaken with bypass surgery if the initial treatment was angioplasty or use of the endovascular device or with surgical revision if the initial treatment was bypass surgery.

Because the procedural risk, cost, and patency curves of the endovascular device are unknown, the following assumptions were made. The patency curve of the endovascular device was assumed to relate, through a proportional hazards model, to that of PTA, which implies that the curves of the endovascular device and of PTA were similar in shape but different in height. The 5-year patency rate was used as a measure for the height of the patency curve. The risk and complications of an endovascular device and the procedural cost, excluding the cost of the device itself, will ideally be approximately the same as those of PTA or lower.

However, since the procedural risk of a hypothetical device is unknown, we assumed that the morbidity, mortality, and convalescence period associated with an endovascular device would be 1.5 times higher than that of PTA. The procedural cost of the device (excluding that of the device itself) was assumed to be approximately the same as that of PTA.

Threshold analysis was performed to determine criteria (ie, combinations of 5 -year patency rates and costs) that would make the device equivalent in terms of cost-effectiveness compared with bypass surgery and PTA. The model was developed from the perspective of the health care system. All costs were adjusted to 1999 U.S. dollars with the use of the medical care-specific consumer price index.

\section{Data and Data Sources}

Estimates of procedural mortality, morbidity, amputation rate, quality-oflife adjustments, costs, and patency rates following femoropopliteal PTA and bypass surgery were based on findings from a published meta-analysis and decision analysis $(4,10)$. The meta-analysis involved a combination of literature data published between 1985 and 1993 and pooled patency results following bypass surgery and PTA for the treatment of femoropopliteal arterial disease, with the use of a method based on the proportional hazards model and the actuarial life-table approach (4). The decision analysis involved a combination of literature data published in 1995 and earlier and an examination of the choice between bypass surgery and PTA for femoropopliteal arterial disease (10).

Table 1 presents the data on bypass surgery and PTA incorporated in the model. The costs for angioplasty and bypass procedures, physician services, noninvasive testing during outpatient follow-up, amputation plus rehabilitation, and annual costs of treatment after an amputation or with major morbidity were based on published and unpublished data on charges from the Brigham and Women's Hospital Vascular Service $(10,11)$. All data on charges were adjusted with the use of cost-to-charge ratios specified by the cost center and the fiscal year.

Quality-of-life adjustments were based on the experience of two vascular surgeons, two interventional radiologists (including M.G.M.H.), and an internist who estimated the various health states related to peripheral arterial disease with the use of an abbreviated form of the Health Utilities Index. This index is used to rate physical function, role function, social and emotional well-being, and general health (10).

The amputation rate following revascularization was assumed to depend on the initial symptomatic status. Each year, on average, $1.2 \%$ of patients with claudication, $2.3 \%$ of patients with rest pain, and $6.4 \%$ of patients with tissue loss underwent amputation $(12,13)$. Of these pa- 
tients, an estimated $11.5 \%$ did not survive the amputation, and another 38\% experienced major morbidity (14-16). The convalescence period following amputation was approximately 82 days (16).

The total cost of amputation, including cost of rehabilitation, was estimated to be $\$ 34,384(17,18)$. Follow-up of patients after revascularization cost, on average, \$543 in the 1st year, \$182 annually thereafter if the artery or bypass remained patent, and \$543 annually if failure occurred (10). The annual cost of long-term care and treatment in patients who underwent amputation of a lower limb was estimated to be $\$ 48,877$ per year $(17,19-21)$. The cost of care and treatment of patients with major morbidity following revascularization or amputation was estimated to be $\$ 11,947$ (22).

The relative risk of overall mortality of patients with peripheral arterial disease was estimated to be 3.1 compared with that of the general population matched for age and sex $(12,23)$. In view of the recommendations of the Panel on Costeffectiveness in Health and Medicine (24), both costs and benefits were discounted at a rate of $3 \%$.

\section{Determination of Criteria and Threshold Analysis}

A strategy was considered cost-effective compared with another if the gain in quality-adjusted life-years (QALYs) justified the additional monetary costs. The trade-off between QALYs and additional monetary cost was considered justified if it did not exceed society's maximum willingness to pay for an incremental gain of 1 QALY.

To facilitate the calculation of the threshold values for patency and cost, the net health benefit (NHB) approach was used to compare the use of the hypothetical endovascular device with currently used interventional strategies (25). The NHB is used to combine costs, QALYs, and an estimate of society's willingness to pay, $\lambda$, for an incremental gain of 1 QALY in one expression. For each strategy, we computed the NHB with the use of the equation NHB = QALYs costs/ $\lambda$ (25). Two strategies were considered equivalent in terms of cost-effectiveness if they yielded the same NHB.

The NHB makes a trade-off between QALYs gained and monetary expense. In essence, the use of the NHB is the same as the use of incremental cost-effectiveness ratios, but technically, the use of NHB is more practical. The difference between the use of the NHB approach and the

TABLE 2

Health Effects, Costs, and NHB of Currently Available Therapies for Femoropopliteal Arterial Disease

\begin{tabular}{lllcc}
\hline Indication and Lesion & $\begin{array}{c}\text { Initial } \\
\text { Treatment }\end{array}$ & $\begin{array}{c}\text { QALE } \\
(\mathrm{QALY})\end{array}$ & $\begin{array}{c}\text { Cost } \\
(\$)\end{array}$ & $\begin{array}{c}\text { NHB (QALY } \\
\text { equivalents) }\end{array}$ \\
\hline $\begin{array}{l}\text { Claudication } \\
\text { Stenosis }\end{array}$ & PTA & 5.85 & 22,758 & 4.71 \\
Stenosis & Bypass & 5.46 & 33,229 & 3.80 \\
Occlusion & PTA & 5.59 & 32,131 & 3.99 \\
$\begin{array}{l}\text { Occlusion } \\
\text { Rest pain }\end{array}$ & Bypass & 5.46 & 33,229 & 3.80 \\
Stenosis & PTA & 5.26 & 42,372 & 3.14 \\
Stenosis & Bypass & 5.00 & 44,694 & 2.76 \\
Occlusion & PTA & 4.83 & 55,074 & 2.08 \\
Occlusion & Bypass & 5.00 & 44,694 & 2.76 \\
Tissue loss & & & & \\
Stenosis & PTA & 5.20 & 48,589 & 2.77 \\
Stenosis & Bypass & 4.92 & 53,346 & 2.25 \\
Occlusion & PTA & 4.74 & 65,578 & 1.46 \\
Occlusion & Bypass & 4.92 & 53,346 & 2.25 \\
\hline
\end{tabular}

Note.-QALE = quality-adjusted life expectancy. All costs are from the perspective of the health care system and include hospital and physician costs for the initial and secondary procedures, treatment of complications, follow-up, long-term care, and amputation and rehabilitation.

incremental cost-effectiveness ratio approach is that in the use of incremental cost-effectiveness ratios, society's willingness to pay can be considered after the results are obtained, whereas in the use of the NHB approach, an estimate of society's willingness to pay must be incorporated in the calculation. For a given estimate of society's willingness to pay, however, the conclusions with the use of either method will be the same. To estimate the effect of the chosen willingnessto-pay value, one can repeat the analysis for a range of values. Published estimates (25) for willingness to pay range from $\$ 20,000$ to $\$ 100,000$ per QALY gained, and we therefore considered this range in our calculations.

\section{Baseline and Sensitivity Analysis}

The baseline case used in the analysis was that of a 65-year-old man with femoropopliteal arterial disease without comorbidity or other risk factors. In our baseline analysis, we assumed society's willingness to pay to be $\$ 20,000$ per QALY gained. In the subgroup analysis, we explored the effect of disease severity (disabling claudication, rest pain, tissue loss) and lesion type (stenosis, occlusion).

In a one-way sensitivity analysis, we explored the effect of varying age (55 and 75 years), sex, society's willingness to pay for the gain of 1 QALY $(\$ 50,000$ and $\$ 100,000)$, and the discount rate $(2 \%$ and $5 \%$ ) on our results. Furthermore, we performed one-way sensitivity analyses to explore the effect of a lower and higher (one and two times that of PTA) procedural risk of the device, including morbidity, mortality, and time lost due to the intervention. We assumed that the morbidity, mortality, and time lost due to the intervention of the endovascular device would not exceed that of bypass surgery. Finally, we explored the effect of varying age, sex, society's willingness to pay, and procedural risk simultaneously in a fourway sensitivity analysis.

\section{RESULTS}

Whereas bypass surgery yielded the highest NHB (Table 2) in patients with chronic critical ischemia (rest pain or tissue loss) and a femoropopliteal occlusion, PTA yielded the highest NHB in stenotic femoropopliteal lesions irrespective of the clinical indication. The Figure presents the target values that would make the use of an endovascular device costeffective compared with currently used procedures. For example, the Figure, part a, shows that an endovascular device that costs $\$ 2,500$ and that is associated with a 5 -year patency rate of $80 \%$ would be costeffective compared with PTA for the treatment of a femoropopliteal stenosis, independent of the clinical indication. However, an endovascular device with the same long-term patency but with a cost of $\$ 7,500$ would not be cost-effective compared with PTA for the treatment of a stenosis and claudication, whereas it would be cost-effective for the treatment of a stenosis and critical ischemia. The Figure, part $b$, shows that the lines are 


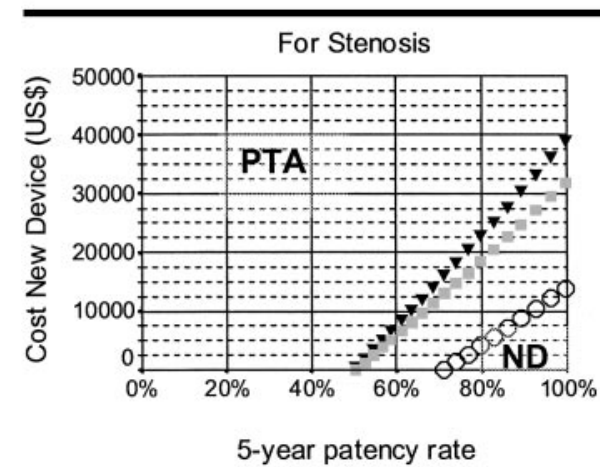

5-year patency rate

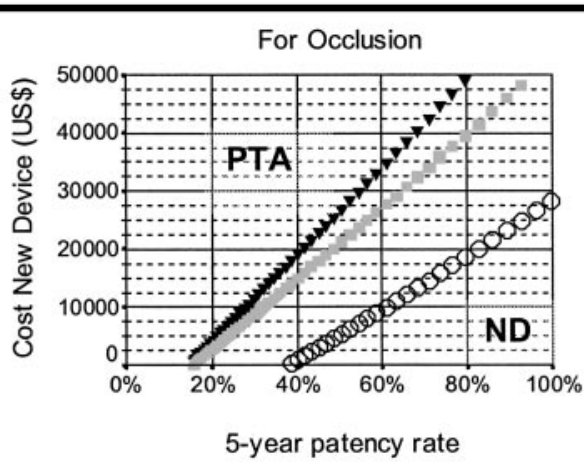

b.

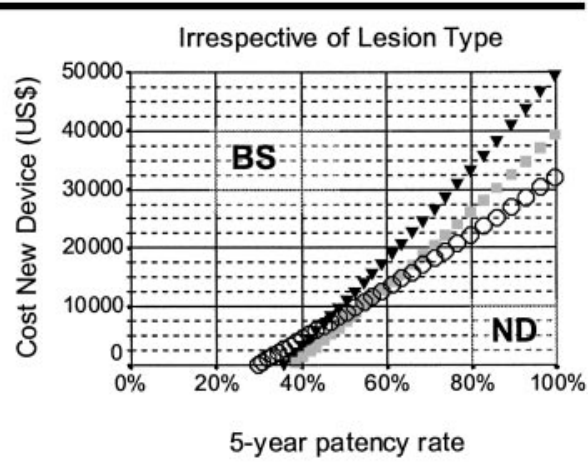

c.

Target values that would make an endovascular device cost-effective compared with (a) angioplasty for a stenosis, (b) angioplasty for occlusions, and (c) bypass surgery. The $\mathrm{x}$ axis represents hypothetical 5-year patency rates of the endovascular device; the y axis, hypothetical costs. Lines represent combinations of 5-year patency rates and costs that would make the endovascular device equivalent to the therapy to which it is being compared in terms of cost-effectiveness, depending on clinical indication: tissue loss $(\boldsymbol{\nabla})$, rest pain (圈), and claudication $(\bigcirc)$. Area below the lines represents the target combinations of patency rates and costs that would make the device (ND) more cost-effective than the therapy to which it is being compared. Area above the lines represents combinations that would make either bypass surgery $(B S)$ or angioplasty $(P T A)$ more cost-effective.

\begin{tabular}{|c|c|c|c|c|}
\hline \multirow[b]{2}{*}{ Procedure and Indication } & \multicolumn{2}{|c|}{ Patency with $\$ 3,000$ Device } & \multicolumn{2}{|c|}{ Patency with $\$ 6,000$ Device } \\
\hline & One Year & Five Year & One Year & Five Year \\
\hline \multicolumn{5}{|l|}{ Bypass surgery } \\
\hline Claudication & $53(41-57)$ & $37(25-41)$ & $59(44-64)$ & $44(28-49)$ \\
\hline Critical ischemia & $58(45-61)$ & $43(29-46)$ & $63(47-68)$ & $48(31-54)$ \\
\hline \multicolumn{5}{|l|}{ PTA for occlusion } \\
\hline Claudication & $60(53-65)$ & $45(36-51)$ & $66(54-73)$ & $52(38-61)$ \\
\hline Critical ischemia & $36(28-39)$ & $20(13-22)$ & $42(29-46)$ & $25(14-29)$ \\
\hline \multicolumn{5}{|l|}{ PTA for stenosis } \\
\hline Claudication & 85 (79-91) & $77(69-86)$ & $89(80-98)$ & $84(70-96)$ \\
\hline Critical ischemia & $69(61-72)$ & $55(46-60)$ & $72(62-78)$ & $60(47-67)$ \\
\hline
\end{tabular}

Note.-Patency rates were determined at baseline analysis. Numbers in parentheses represent the range of patency rates found after performing a four-way sensitivity analysis with simultaneously varying sex, age $(55,65$, and 75 years), society's willingness to pay $(\$ 20,000, \$ 50,000$, and $\$ 100,000$ per QALY), and procedural risk of the new device $(1,1.5$, and 2 times that of PTA).

more to the left compared with those in part a, which implies that for the treatment of occlusions, the target 5-year patency rates are less stringent.

By considering the treatment of critical ischemia and an occlusion, the graphs show that for any given cost of the endovascular device, the patency rates needed to make use of the device cost-effective compared with bypass surgery (Fig 1, part c) were higher than those required to make the device cost-effective compared with PTA (Figure, part b). This is consistent with the findings presented in Table 2 , which shows that bypass surgery yielded a higher NHB than PTA in the treatment of critical ischemia and occlusion.

A striking finding was the high acceptable cost for an endovascular device, provided that it is associated with a high long-term patency rate. For example, the Figure, part c, shows that, if an endovascular device has a 5-year patency rate of $80 \%$, the cost of the device may increase to $\$ 20,000$, and it would still be costeffective compared with bypass surgery, irrespective of the clinical indication.

The results are shown in more detail in Table 3 for two endovascular devices, one device that costs $\$ 3,000$ and a second device that costs $\$ 6,000$. For clarity, rest pain and tissue loss were replaced by critical ischemia to take into account the highest patency rate required. Table 3 shows the 5 -year patency rates and the associated 1 -year patency rates that would make an endovascular device equivalent in terms of cost-effectiveness compared with currently used procedures. For example, the 5-year patency rate that would make a device that costs $\$ 3,000$ cost-effective compared with bypass surgery for the treatment of critical ischemia was $43 \%$ in the baseline analysis.

Results of one-way sensitivity analyses demonstrated that with an increase in society's willingness to pay, female sex, and a younger age, lower patency rates would be acceptable for an endovascular device. A higher procedural risk and a higher age increased the required patency rates. The tabulated ranges (Table 3 ) indicate the lowest and highest required patency rates found when age, sex, procedural risk, and society's willingness to pay were simultaneously varied in a sensitivity analysis. Variation of the discount rate from $2 \%$ to $5 \%$ in one-way sensitivity analyses resulted in an absolute difference of, at most, $1 \%$ when these rates were compared with the 5 -year patency rates found in the baseline analyses.

\section{DISCUSSION}

We report target values of primary patency rates and costs that a hypothetical endovascular device for the treatment of femoropopliteal arterial disease would have to attain to be cost-effective compared with currently used therapies. The results help predict under what conditions an endovascular device would be the most promising and can help focus future technologic development of endovascular devices. As previously demonstrated (10), the results illustrate that when currently used procedures are considered, PTA is more cost-effective compared with bypass surgery in the treatment of milder forms of femoropopliteal arterial disease, and bypass surgery is the 
treatment of choice in more severe disease.

When an endovascular device is considered, the results suggest that the target 5 -year primary patency rate that would make a device that costs $\$ 3,000$ equivalent in terms of cost-effectiveness compared with bypass surgery ranges from $25 \%$ to $46 \%$. The 5-year patency rates that would make a device that costs $\$ 3,000$ equivalent in terms of cost-effectiveness compared with PTA in the treatment of claudication and femoropopliteal stenosis ranged from $69 \%$ to $86 \%$. Furthermore, the results suggest that use of a hypothetical endovascular device, with a 5 -year patency rate of $80 \%$, would be cost-effective compared with bypass surgery, even if the device costs up to $\$ 20,000$, irrespective of clinical indication.

Caution should be exercised when conclusions are made about cost-effectiveness. Cost-effectiveness is always relative. In the current study, we compared treatment with use of an endovascular device with bypass surgery and with PTA. If more than one endovascular device were to be made available on the market, they would have to be compared with each other. Thus, an expensive currently available device may have a cost-effectiveness ratio just below the willingnessto-pay threshold compared with bypass surgery or with PTA, whereas in the future, that device may no longer be costeffective if another device yields nearly the same effectiveness at a lower cost.

Limitations of the analysis lie within the assumptions of the model. First, we did not update the data concerning the currently used procedures in the model. Although the patency rates and risks of both PTA and bypass surgery are continually improving, a major improvement in the past 5 years is unlikely (1). Therefore, we assumed that the data used in the 1995 model were still valid. Furthermore, there is evidence that the cost of femoropopliteal revascularization has not changed considerably during the past few years (5).

Second, the data on costs associated with current therapies were collected in a teaching hospital in the United States. Caution should be exercised when the results are generalized to nonteaching hospitals or hospitals in other countries.

Third, we assumed that the procedural risk (complications, mortality) of an endovascular device would be 1.5 times higher than that of PTA. We assumed this because, ideally, the procedural risk of an endovascular treatment would be the same as that of PTA or lower. However, to make the baseline results also applicable for an endovascular treatment associated with less favorable morbidity and mortality rates, we assumed that the procedural risk associated with the endovascular device was 50\% higher than that of PTA. In sensitivity analyses, we explored the effect of varying the procedural risk and the convalescence period, ranging from one to two times that of PTA, and found that an increase in procedural risk and convalescence period resulted in more stringent target values for the endovascular device.

Fourth, the hypothetical patency curves of the endovascular device were based on the patency curve of PTA. By doing this, we assumed that most failures occur during the 1st year after the procedure. We assumed this because both are endovascular treatments, but the true curve is unknown.

Fifth, the choice of the threshold value of society's willingness to pay may have been a limitation. Estimates of society's willingness to pay are highly dependent on the societal and decision context (26). In the baseline analyses of the current study, we assumed society's willingness to pay to be $\$ 20,000$ per QALY gained, and we explored the influence of choosing a threshold of $\$ 50,000$ per QALY gained and that of $\$ 100,000$ per QALY gained on our results. Because we chose a higher threshold, which implies the acceptance of higher costs for the same health effect, the results yielded less stringent criteria for the hypothetical endovascular device.

Current research on endovascular devices to treat femoropopliteal arterial disease focuses on the problem of intimal hyperplasia, which causes secondary obstruction after an angioplasty procedure or stent implantation. The idea is to prevent secondary obstruction by covering the arterial wall with prosthetic material, such as polytetrafluoroethylene, now often used as the conduit material in bypass surgery. Primary patency rates reported (27-29) thus far associated with such stent-grafts range from $73 \%$ at 12 months to $59 \%$ at 18 months to $46 \%$ at 24 months. One group of investigators (30) recently reported less favorable results (12-month patency rate, 29\%). The results in the current analysis suggest that the target 12-month primary patency rate for an endovascular device that costs $\$ 3,000$ is approximately $41 \%$ $61 \%$. This finding suggests that, if the assumptions in this study hold true, the use of these devices may already be cost- effective compared with bypass surgery. The validity and generalizability of the reported patency rates in these initial studies were, however, low because the sample sizes were limited and because data on long-term results were not available.

The methods used in this study can be applied to many situations where investigators are developing therapeutic technologies. Other examples of new technologies under development are endovascular devices for the treatment of abdominal aortic aneurysms and catheter techniques with the use of coiling devices for the treatment of cerebral aneurysms. Perhaps the first step in investigations of new technologies, both therapeutic and diagnostic, should be to establish clear-cut goals for the performance of such technologies compared with existing ones. Such analyses could help focus research and the development of new technologies and thereby help to save valuable resources $(8,9)$.

In conclusion, the results of this costeffectiveness analysis in which target cost and patency rates were estimated demonstrate that there is a place for a new endovascular therapy in the treatment of femoropopliteal arterial disease. The target values of patency rates and costs determined in this study that would make the use of an endovascular device costeffective compared with the currently used therapies are probably attainable, and further development of endovascular devices seems warranted.

\section{References}

1. Dormandy JA, Rutherford RB. Management of peripheral arterial disease (PAD): TASC Working Group. J Vasc Surg 2000; 31:S1-S296.

2. Stanley B, Teague B, Raptis S, Taylor DJ, Berce M. Efficacy of balloon angioplasty of the superficial femoral artery and popliteal artery in the relief of leg ischemia. J Vasc Surg 1996; 23:679-685.

3. Jeans WD, Cole SE, Horrocks M, Baird RN. Angioplasty gives good results in critical lower limb ischaemia: a 5-year follow-up in patients with known ankle pressure and diabetic status having femoropopliteal dilations. Br J Radiol 1994; 67:123128.

4. Hunink MGM, Wong JB, Donaldson MC, Meyerovitz MF, Harrington DP. Patency results of percutaneous and surgical revascularization for femoropopliteal arterial disease. Med Decis Making 1994; 14: 71-81.

5. Jansen RM, de Vries SO, Cullen KA, Donaldson MC, Hunink MG. Cost-identification analysis of revascularization procedures on patients with peripheral arterial occlusive disease. J Vasc Surg 1998; 28: 617-623.

6. Vroegindeweij D, Vos LD, Tielbeek AV, Buth J, van den Bosch HC. Balloon angio- 
plasty combined with primary stenting versus balloon angioplasty alone in femoropopliteal obstructions: a comparative randomized study. Cardiovasc Intervent Radiol 1997; 20:420-425.

7. Do-dai-Do, Triller J, Walpoth BH, Stirnemann P, Mahler F. A comparison study of self-expandable stents vs balloon angioplasty alone in femoropopliteal artery occlusions. Cardiovasc Intervent Radiol 1992; 15:306-312.

8. Phelps CE, Mushlin AI. Focusing technology assessment using medical decision theory. Med Decis Making 1988; 8:279289.

9. Hunink MG, Kuntz KM, Fleischmann KE, Brady TJ. Noninvasive imaging for the diagnosis of coronary artery disease: focusing the development of new diagnostic technology. Ann Intern Med 1999; 131:673-680.

10. Hunink MGM, Wong JB, Donaldson MC, Meyerovitz MF, de Vries JA, Harrington DP. Revascularization for femoropopliteal disease: a decision and cost-effectiveness analysis. JAMA 1995; 274:165-171.

11. Hunink MGM, Cullen KA, Donaldson MC. Hospital costs of revascularization procedures for femoropopliteal arterial disease. J Vasc Surg 1994; 19:632-641.

12. Howell MA, Colgan MP, Seeger RW, Ramsey DE, Sumner DS. Relationship of severity of lower limb peripheral vascular disease to mortality and morbidity: a sixyear follow-up study. J Vasc Surg 1989; 9:691-697.

13. McDaniel MD, Cronenwett JL. Basic data related to the natural history of intermittent claudication. Ann Vasc Surg 1989; 3:273-277.
14. Plecha FR, Bertin VJ, Plech EJ, et al. The early results of vascular surgery in patients 75 years of age and older: an analysis of 3259 cases. J Vasc Surg 1985; 2:769-774.

15. Miller N, Dardik H, Wolodiger F, et al Transmetatarsal amputation: the role of adjunctive revascularization. J Vasc Surg 1991; 13:705-711.

16. Weiss GN, Gorton A, Read RC, Neal LA. Outcomes of lower extremity amputations. J Am Geriatr Soc 1990; 38:877-883.

17. Mackey WC, McCullough JL, Conlon TP et al. The costs of surgery for limb-threatening ischaemia. Surgery 1986; 99:26-35.

18. Raviola CA, Nichter LS, Baker JD, Busuttil RW, Machleder HI, Moore WS. Cost of treating advanced leg ischemia. Arch Surg 1988; 123:495-496.

19. Gupta SK, Veith FJ, Ascer E, White Flores SA, Gliedman ML. Cost factors in limbthreatening ischaemia due to infrainguinal arteriosclerosis. Eur J Vasc Surg 1988; 2:151-154.

20. Cheshire NJW, Wolfe JHN, Noone MA, Davies L, Drummond M. The economics of femorocrural reconstruction for critical leg ischemia with and without autologous vein. J Vasc Surg 1992; 15:167-175.

21. Callow AD, Mackey WC. Costs and benefits of prosthetic vascular surgery. Int Surg 1988; 73:237-240.

22. Wittels EH, Hay JW, Gotto AM. Medical costs of coronary artery disease in the United States. Am J Cardiol 1990; 65:432440.

23. Criqui $\mathrm{MH}$, Langer $\mathrm{RD}$, Fronek $\mathrm{A}$, et al. Mortality over a period of 10 years in patients with peripheral arterial disease. N Engl J Med 1992; 326:381-386.
24. Gold MR, Siegel JE, Russell LB, Weinstein MC. Time preference. In: Gold MR, Siegel JE, Russell LB, Weinstein MC, eds. Costeffectiveness in health and medicine. 1st ed. New York, NY: Oxford University Press, 1996; 214-235.

25. Stinnett AA, Mullahy J. Net health benefits: a new framework for the analysis of uncertainty in cost-effectiveness analysis. Med Decis Making 1998; 18:S68-S80.

26. Gold MR, Siegel JE, Russell LB, Weinstein MC. Reporting cost-effectiveness studies and results. In: Gold MR, Siegel JE, Russell LB, Weinstein MC, eds. Cost-effectiveness in health and medicine. 1st ed. New York, NY: Oxford University Press, 1996; 277-303.

27. Spoelstra H, Casselman F, Lesceu O. Balloon-expandable endobypass for femoropopliteal atherosclerotic occlusive disease: a preliminary evaluation of fifty-five patients. J Vasc Surg 1996; 24:647-654.

28. Henry M, Amor M, Cragg A, et al. Occlusive and aneurysmal peripheral arterial disease: assessment of a stent-graft system. Radiology 1996; 201:717-724.

29. Rodriguez-Lopez JA, Soler L, Werner A, Martinez E, Papazoglou K, Diethrich EB. Long-term follow-up of endoluminal grafting for aneurysmal and occlusive disease in the superficial femoral artery. J Endovasc Surg 1999; 6:270-277.

30. Kessel DO, Wijesinghe LD, Robertson I, et al. Endovascular stent-grafts for superficial femoral artery disease: results of 1-year follow-up. J Vasc Interv Radiol 1999; 10: 289-296. 